\title{
Laboratory Data Integration into Medical Record
}

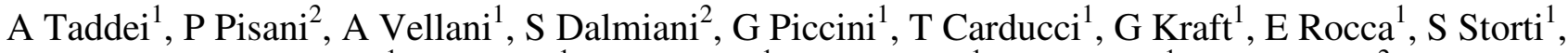 \\ A Andrenelli ${ }^{1}, \mathrm{MS}_{\text {Parri }}{ }^{1}, \mathrm{M}_{\text {Cardillo }}{ }^{1}, \mathrm{P}_{\text {Lauciello }}{ }^{1}, \mathrm{~S}_{\text {Gwynne }}{ }^{1}$, A Macerata $^{2}$ \\ ${ }^{1}$ CNR Institute of Clinical Physiology, G Pasquinucci Hospital, Massa, Italy \\ ${ }^{2} \mathrm{CNR}$ Institute of Clinical Physiology, Pisa, Italy
}

\begin{abstract}
Laboratory Information System, integrated with the Hospital Information System, has been developed at the G.Pasquinucci Hospital, section of Institute of Clinical Physiology of National Research Council (CNR), specialized in adult and paediatric cardiac surgery. The aim was to automate the testing process from clinical departments to laboratory and back into medical record. Laboratory workflow consists of three parts: (a) test ordering by clinical staff, printing bar-coded ID labels and transmitting orders by network to laboratory; (b) processing test requests and controlling identified specimens by laboratory staff, providing work orders to analytical instruments and validation of results authorizing delivery into the hospital clinical repository; (c) consulting test results in clinical departments by referring physicians through the electronic medical record. This year the system has been used on adult patients processing 135000 laboratory tests concerning chemistry, haematology, coagulation and immunology.
\end{abstract}

\section{Introduction}

In health-care laboratory-test results are largely used routinely for supporting physicians to confirm a suspected diagnosis, to monitor effects of treatment or to assess prognosis [1]. The primary function of clinical laboratories is to acquire, validate, interpret and communicate information derived from analysis of patient specimens. Quality of laboratory services depends not only on accuracy and precision of test results, but also on the timeliness of delivery. In hospitals several millions of tests are performed each year. Increasing laboratory workload promoted application of computers since many years ago. Today Laboratory Information Systems (LIS) are used in most hospitals for supporting both data processing and laboratory management. Advanced analytical instruments for automated processing of large series of tests are available, equipped with on-line interface for uploading work orders and providing test results. Various laboratories are distinguished, each handling different types of specimens and using different analytical techniques; typical specialties include clinical chemistry performing chemical analysis of samples, haematology performing tests on cellular elements of blood, immunology measuring antigens and antibodies in body fluids.

Testing process is divided into two cycles: one taking place in clinical departments or ambulatories (first, test ordering and specimens collection, and at last result reporting) and the other within the laboratory (analysis and validation) (figure 1).

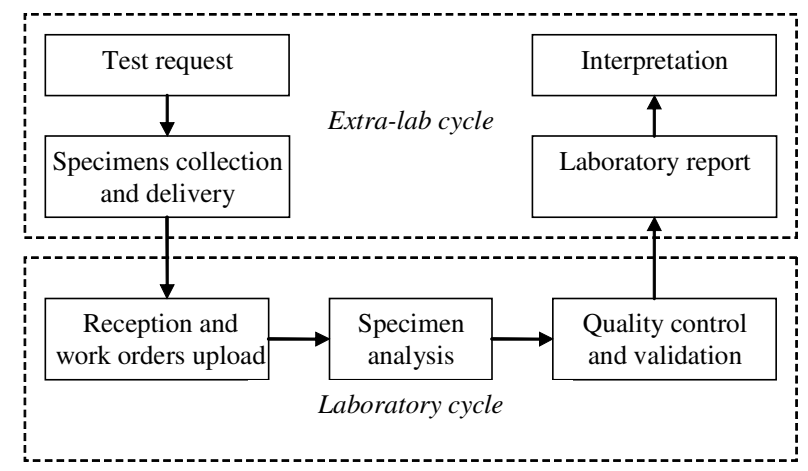

Figure 1 The testing process

The classical way to order a laboratory test for a patient is to fill in a request (often mark-sense form) on which test parameters are pre-printed together with patient identification [2]. The form is sent with the patient's specimens to laboratory where test requests are entered into LIS, either automatically (by optical reader in case of mark-sense forms) or manually. If LIS is linked to Hospital Information System (HIS) (according to IHE) [3] it is possible to request, directly from departments, laboratory tests, even by Electronic Medical Record (EMR). Test requests can be transmitted through network to the LIS, just waiting specimen arrival in the laboratory. In the latter approach patient data are automatically provided from HIS to LIS, as well as identification labels for each specimen container. Work lists are generated with position assignment of specimen containers in trays. In case of modern instruments bar-coded labels can be 
used for enabling automated identification of specimens. Test results are subject to validation by the laboratory staff and then authorized for reporting to the requesting clinician. At the Institute of Clinical Physiology (IFC) (health care institute of National Research Council, specialized in study and treatment of cardiovascular pathologies) HIS development was initiated ten years ago by a national project (SPERIGEST) [4]. Today full integration of clinical and administrative information over a networked structure has been achieved; access to patient information is made available through EMR [5,6]. In this framework a LIS, integrated with HIS, was developed for the automation of workflow of clinical laboratory testing. and printing bar-coded ID labels, while in the laboratory commercial software (LABITUP, Sisge) was applied for data exchange with analyzers. The LIS was linked with the HIS allowing identification of patients at nursing workstation and reporting test results into electronic medical record (EMR). Graphical applications, accessing both LIS and HIS database servers (SQL IBM DB2), were previously developed in MS-C language under IBM OS2 by one of the authors (Paolo Pisani) for use at IFC in Pisa [5]. These programs were modified and extended for application in the departments of G.Pasquinucci Hospital. Automated analytical instruments, charged of most routinely performed tests, were interfaced with the

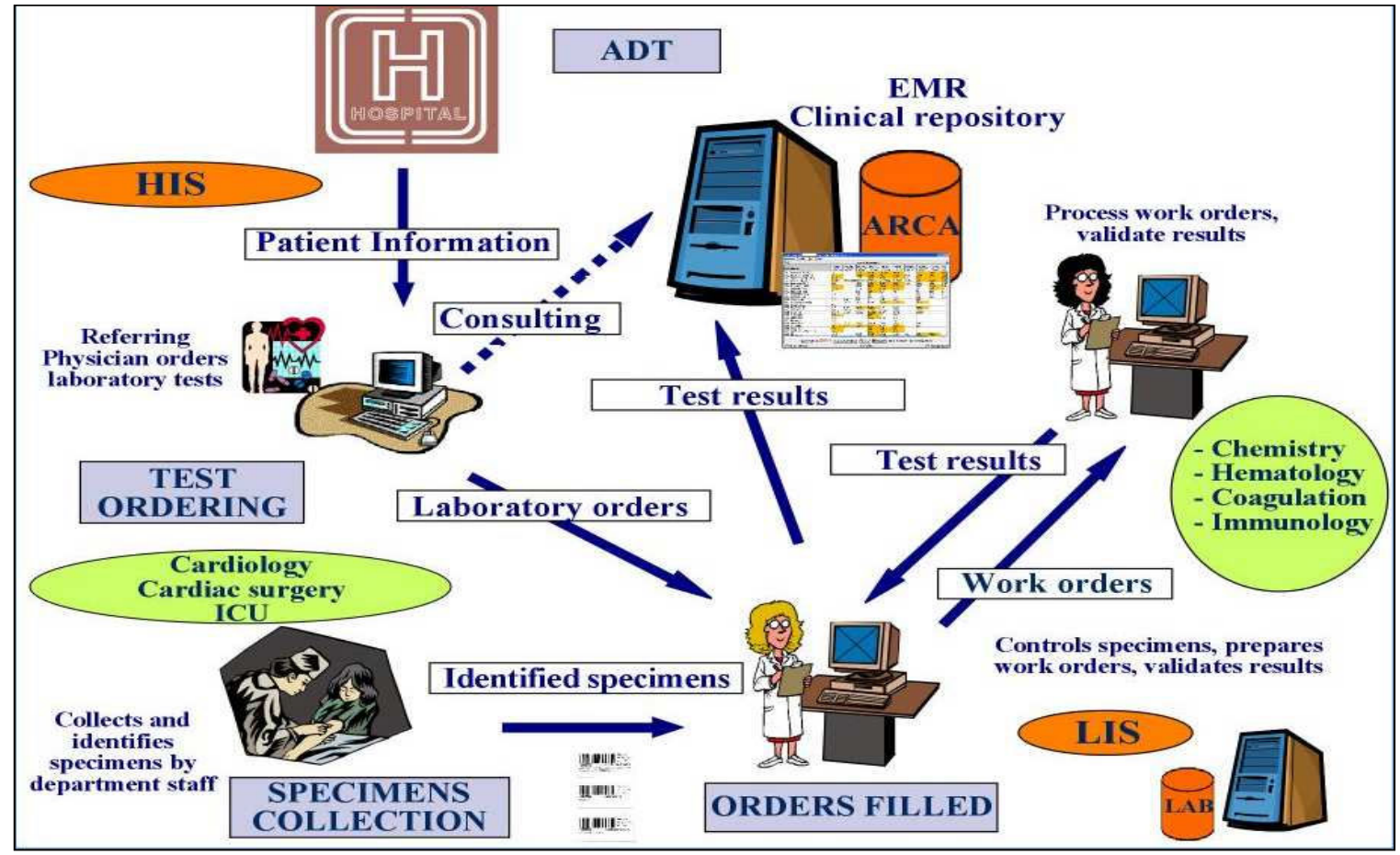

Figure 2 Integrated laboratory testing workflow

The aim was to automate test ordering and collection of identified specimens from clinical departments or ambulatories, providing work orders to analyzers and getting back results, finally transmitted, after validation and authorization, into patient EMR (figure 2). This system was first developed and applied at the head of IFC in Pisa and recently was adapted for use at the institute section in Massa (G.Pasquinucci Hospital), specialized in adult and paediatric cardiac surgery.

\section{Methods}

The LIS, developed at G.Pasquinucci Hospital, is based on a client-server networked architecture. Nursing workstations were set up in the clinical departments (Cardiology, Cardiac Surgery and ICU) for test ordering
LIS; they concern clinical chemistry (Beckman CX), immunology (Abbot Axsym), coagulation (Dasit Sysmex CA-1500) and hematology (Dasit SF 3000). Other few tests (usually performed outside the hospital) continue to be managed manually, actually requiring clinical staff to enter corresponding results directly into EMR to complete laboratory test data needed routinely for patient health care.

The scenario includes the following tasks (figure 2):

- Management of test requests: ordering tests, collection and labeling of sample specimens.

○ Laboratory processing: work orders for analysis of specimens and validation/authorization of results

- Storing results into the clinical repository for consultation by clinical staff through EMR. 


\subsection{Management of test requests}

Laboratory test requests, prescribed by physicians in the care department, are entered in clinical departments by nursing staff using the "order placer" application. First step is the selection of the single patient from the list of patients admitted in the department (retrieved by HIS database through SQL query). In case patient admission has not yet been recorded by administration (ADT), a temporary record is created. Next step is the selection of tests from the full list or, more simply, from "batteries" of tests, routinely requested in common situations (e.g. $1^{\text {st }}$ day admission, post-surgery).

\begin{tabular}{|c|c|c|c|c|c|}
\hline code & dest & test name & color & range norm. & grouping \\
\hline 202 & $F^{*} 111$ & Transaminasi G.O. (AST) & $M$ & $02 \sim$ IU/L $0-50 \sim$ & $-01-02-11-$ \\
\hline 204 & $F^{*} 111$ & Fosfatasi Alcalina (ALP) & M & $02 \sim \mathrm{IU} / \mathrm{L} \sim 42-121 \sim$ & $-01-02-51-$ \\
\hline 205 & $F^{*} 111$ & Latticodeidrogenasi (LD-P) & $\mathbf{M}$ & $02 \sim 1 \mathrm{IU} / \mathrm{L} \sim 266-500 \sim$ & $-01-02$ \\
\hline 502 & $F^{*} 151$ & Digossina & 1 & $02 \sim \mathrm{ng} / \mathrm{mL} \sim 0.3-2.0 \sim$ & $-32-$ \\
\hline 996 & $F^{*} 151$ & Vancomicina & 1 & $02 \sim \mathrm{ng} / \mathrm{ml} \sim 30-40 \sim$ & $-32-$ \\
\hline
\end{tabular}

Table 1 Reference test definition

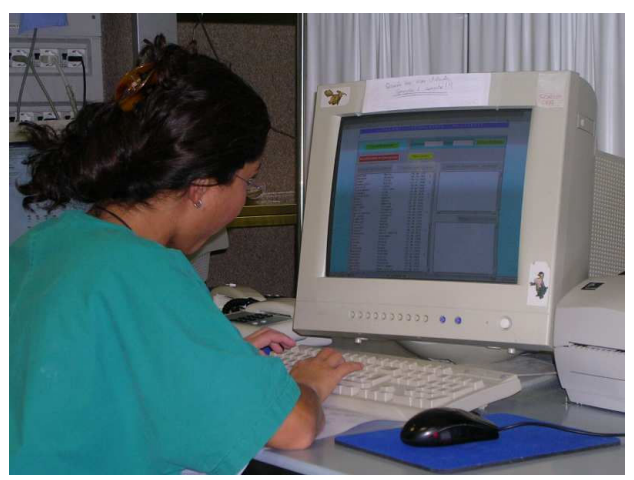

Figure 3 Test ordering by nursing staff

Tables with relations were defined in the LIS database to characterize objects relating to testing process. Tests currently performed in the laboratory (113) are defined by a reference table, in which each test is identified by a key code and characterized in terms of destination laboratory analyzer, color of tube, range of normal values, group assignment, printing order and format (Table 1). Each single request is recorded in the table of requests and the series of tests (coded according to reference table) are recorded in the related table of requested tests.

Each time any request with the selected tests has been recorded through the nursing workstation into the LIS database, automated retrieval from the laboratory workstations is enabled. Actually requests are filtered according to specific destination, as defined in the reference table (e.g. see $\mathrm{F}^{*} 111$ or $\mathrm{F}^{*} 151$ in table 1: the former corresponding to chemical chemistry and the latter to immunology), and distributed to the corresponding analytical instrument.

Lists of the requested tests and bar-coded labels are

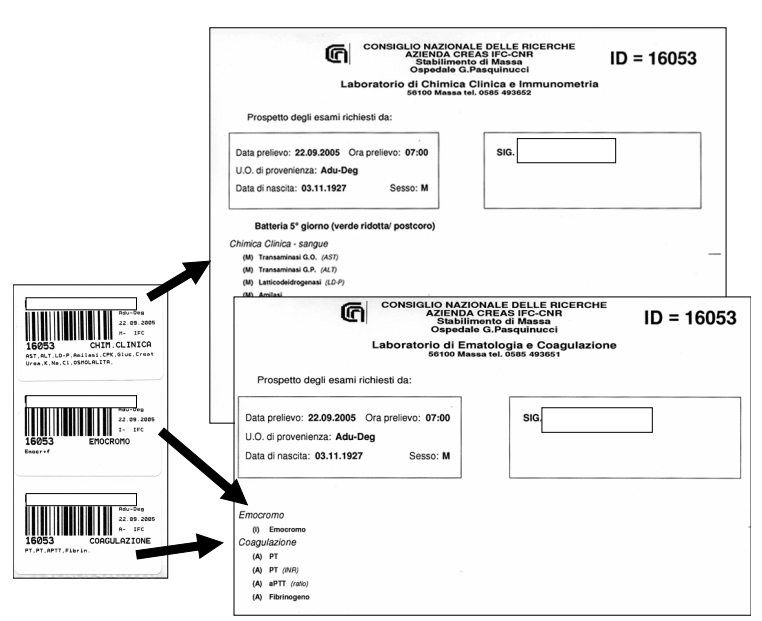

Figure 4 ID bar-coded labels and test orders printed with the patient's name, unique identification

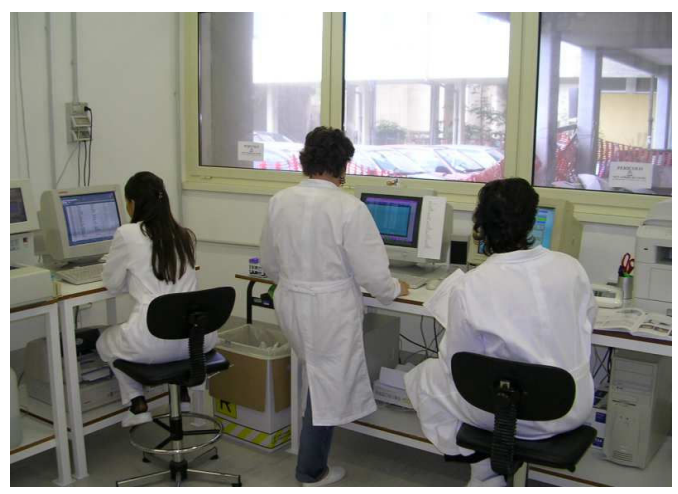

Figure 5 Laboratory staff controlling test requests, preparing work orders and validating

number (ID), department and date of collection for each individual specimen container (figure 4). A proper number of labels and requests are provided depending on how many types of tests (performed on different analyzers) are requested or from the quantity of tests of the same type. Specimen tubes, identified by ID labels, are finally transferred to the laboratory.

\subsection{Laboratory processing}

The sequencing of material flow (the specimens) and the electronic flow (the test request) is not necessarily synchronized. The laboratory staff looks at each request using "order filler" application and ensures that all required specimens are available and conform to the order (figure 5). The order is rejected or accepted with modifications if needed. The order is then confirmed and scheduled by "order filler". Each order is splitted into one or more work orders, either (the majority) sent to the corresponding automated analyzers (chemistry, immunology, coagulation or hematology) or manually 
managed by laboratory staff. Identified specimen tubes are distributed to the analyzers according to the work orders and uploaded in the trays in the positions assigned by application program. Tubes can be positioned randomly if bar code recognition has been enabled. Then the laboratory staff performs a validation (technical and clinical) of all the results returned from analyzers and finally authorizes only the right ones, which are recorded into the clinical repository (ARCA) of medical records. Reports are finally printed and signed by laboratory head.

\subsection{Reporting results in EMR}

Results of laboratory tests, managed by LIS and recorded in clinical repository (ARCA), are reported for consultation in the EMR, which is routinely used in the hospital for patient care. Test results are represented in

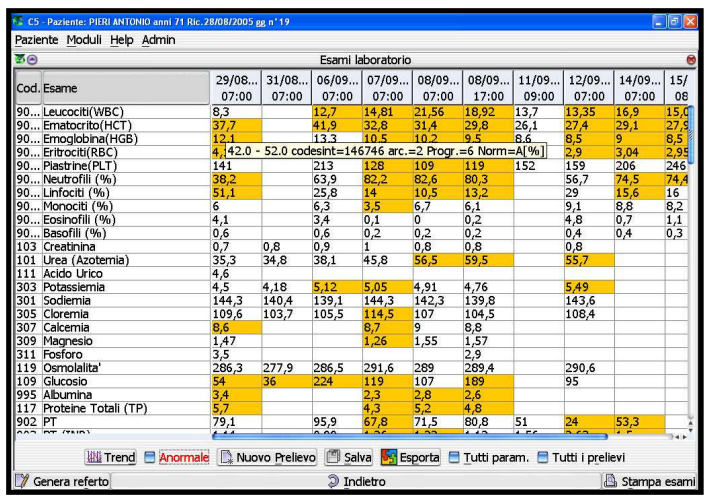

Figure 6 Reporting test results in EMR

tabular form where abnormal values are marked for evidence and the applied normality ranges are made available. Graphical trends of parameters can be represented for analysis of time variations. PDF printouts are provided for documentation of patient medical record.

\section{Results}

Since January 2005 the system has been used routinely for automating flow of laboratory data on adult patients admitted in departments of Cardiology, Cardiac Surgery and ICU. Up to 5500 requests have been processed for a total amount of 135000 tests (out of 113 types). Recently application to pediatric patients (Cardiology/Cardiacsurgery and ICU) has been scheduled.

\section{Discussion and conclusions}

Both clinical and laboratory staff, after initial training and efforts, have got advantages using the system in terms of both time saving and reliable documentation. The nursing staff found the pre-printed labels very useful and effective. Reports of test values in EMR were effective for patient care by physicians in clinical departments. Increasing laboratory workload with the expected extension of laboratory service to outpatients will further benefit by the automation of workflow. Given IHE initiatives [3] for standardization of laboratory integration in health care enterprises using HL7 protocols, a revision of the actual system is scheduled to be eventually upgraded for improving performance.

\section{Acknowledgements}

We gratefully acknowledge advice of physicians and nursing personnel at G.Pasquinucci Hospital and of colleagues at IFC in Pisa: D.Pierotti, (database); L.Landucci (network), A.Ciriegia (operating systems).

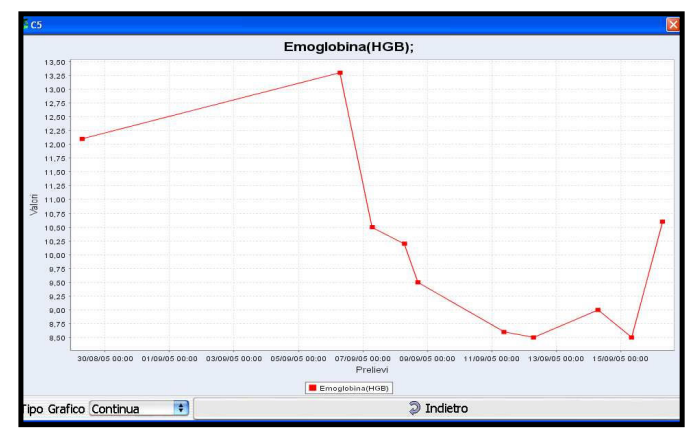

Figure 7 Plotting parameter time variations

\section{References}

[1] Smith JW, Svirbely JR. Laboratory information systems. In: EH Shortliffe, LE Perreault, editors. Medical Informatics: computer applications in health care. Reading, MA, USA: Addison-Wesley Publishing Company, 1990: 273-297.

[2] Van Bemmel JH, Musen MA. Handbook of medical informatics. Houten (NL): Bohn Stafleu Van Loghum, 1997: 207-17.

[3] http://www.ihe-europe.org/ IHE laboratory technical framework, vol.1, rev.1.1, 2004

[4] Macerata A, Landucci L et al. Networking for health care administration and delivery: the information system at the CNR Institute of Clinical Physiology. In: Proceedings of Health Telematics '95. Pisa: CNR, 1995, 113-18.

[5] Carpeggiani C, Dalmiani S, Taddei A et al. Use of an electronic medical record in a department of Cardiology. Computers in Cardiology 2000; 27: 291-294.

[6] Taddei A, Dalmiani S, Piccini G et al. Data integration in cardiac surgery and resource management. Computers in Cardiology 2003; 30:279-282.

Address for correspondence

Alessandro Taddei

CNR Institute of Clinical Physiology

Hospital Pasquinucci - Medical Informatics

Via Aurelia Sud - 54100 Massa (Italy)

taddei@ifc.cnr.it 